\title{
Ether-a-go-go related gene-1a potassium channel abundance varies within specific skeletal muscle fiber type
}

\author{
Luke B. Anderson (1), Chase D. Latour (2), Omar Khader (1), Bryce H. Massey (3), \\ Brittan Cobb (1), Amber L. Pond (1) \\ (1) Department of Anatomy, Southern Illinois University School of Medicine, Carbondale, IL; \\ (2) Department of Psychiatry, Washington University in St. Louis, St. Louis, MO; (3) Brown \\ University, Providence, RI, USA
}

This article is distributed under the terms of the Creative Commons Attribution Noncommercial License (CC BY-NC 4.0) which permits any noncommercial use, distribution, and reproduction in any medium, provided the original author(s) and source are credited.

\begin{abstract}
The ERG1A $\mathrm{K}^{+}$channel, which is partially responsible for repolarization of the cardiac action potential, has also been reported in skeletal muscle where it modulates ubiquitin proteolysis. Because ERG1A protein appears variably expressed in muscles composed of mixed fiber types, we hypothesized that its abundance in skeletal muscle might differ with fiber type. Indeed, skeletal muscle fibers vary in speed of contraction (fast or slow), which is mainly determined by myosin heavy chain (MyHC) isoform content, but a sarcolemmal $\mathrm{K}^{+}$channel might also modulate contraction speed. To test our hypothesis, we cryo-sectioned Soleus (SOL), Extensor Digitorum Longus (EDL), and Gastrocnemius muscles from five rats. These muscles were chosen because the SOL and EDL contain an abundance of slow- and fast-twitch fibers, respectively, while the Gastrocnemius has a more heterogeneous composition. The muscle sections were co-immunostained for the ERG1A protein and either the fast- or slow-twitch MyHC to identify fiber type. ERG1A fluorescence was then measured in the sarcolemma of each fiber type and compared. The data reveal that the ERG1A protein is more abundant in the fibers of the SOL than in the EDL muscles, suggesting ERG1A may be more abundant in the slow than the fast fibers, and this was confirmed with immunoblot. However, because of the homogeneity of fiber type within these muscles, it was not possible to get enough data from both fiber types within a single muscle to compare ERG1A composition within fiber type. However, immunohistochemistry of sections from the fiber type heterogeneous Gastrocnemius muscle reveals that slow fibers had, on average, a $17.2 \%$ greater ERG1A fluorescence intensity than fast fibers $(p<0.03)$. Further, immunoblot reveals that ERG1A protein is $41.6 \%$ more abundant $(\mathrm{p}=0.051)$ in old than in young rat Gastrocnemius muscle. We postulate that this membrane bound voltage-gated channel may affect membrane characteristics, the duration of the action potential generated, and/or the speed of contraction. Indeed, ERG1A protein is more abundant in aged and atrophic skeletal muscle, both of which exhibit slower rates of contraction.
\end{abstract}

Key Words: Ether-a-go-go related gene, skeletal muscle, atrophy, muscle fiber type.

Skeletal muscle comprises $35-45 \%$ of the human body mass and is critical for movement, posture, and body temperature regulation. Skeletal muscle is composed of muscle fibers that are made up of contractile threads called myofibrils. These myofibrils are composed of repetitive units known as sarcomeres. Each sarcomere is bordered by a Z-disk on each end with an area in the center composed of proteins that make up the M-line. The thick filaments that extend outward from the M-line are composed mainly of both myosin heavy (MyHC) and light chains. The thin filaments, which are connected to the Z-disk by anchor proteins (e.g., titin), are mainly composed of actin filament proteins. Each actin filament is wrapped by tropomyosin proteins, which have troponin protein complexes attached to them. Calcium ions bind to the troponin proteins and this binding causes the tropomyosin protein to change shape, exposing sites on the actin to which the MyHCs can bind. The MyHC heads bind to the actin proteins, forming cross bridges. When activated by ATP, the neck of the myosin head tilts, pulling the Z-disks toward the M-line. The myosin then 
releases from the thin filament, bends back to a resting state, and is ready to bind and contract again if needed. Skeletal muscle fibers vary in their speed of contraction (fast or slow), resistance to fatigability (high or low), and main metabolic pathway for energy production (aerobic or anaerobic). They are generally referred to in terms of speed of contraction such that the main fibers described are slow-twitch (Type 1) or fast-twitch fibers (Type 2A, $2 \mathrm{X}$, and $2 \mathrm{~B}$ ). Other fibers have been described, but those are generally muscle specific and/or not abundant (Table 1). It has been shown that the speed of shortening of unloaded fibers ${ }^{1}$ and the speed of the force transient of fibers under isometric conditions ${ }^{2}$ is mainly determined by the isoform of the myosin heavy chain. Slow-twitch fibers generally contain the MyHC- $\beta$ protein which is encoded by the $M Y H 7$ gene. Fibers with this protein produce ATP mainly by aerobic metabolism and are comparatively more resistant to fatigue. Fast contracting fibers mainly contain one or more of the fast $\mathrm{MyHC}$ proteins: MyHC-2A, MyHC-2B, or MyHC-2X. Fast fibers vary in speed of contraction from $2 \mathrm{~A}, 2 \mathrm{X}$, to $2 \mathrm{~B}$ with $2 \mathrm{~A}$ being the slowest and $2 \mathrm{~B}$ being the fastest. They also vary in terms of their main form of metabolism used to produce ATP, with $2 \mathrm{~A}$ predominantly relying upon aerobic metabolism and $2 \mathrm{X}$ and $2 \mathrm{~B}$ relying predominantly upon the anaerobic pathway. Consequently, $2 \mathrm{~A}$ is more fatigue resistant than $2 \mathrm{X}$, and both $2 \mathrm{~A}$ and $2 \mathrm{X}$ are more fatigue resistant than $2 \mathrm{~B} .^{3,4}$ Muscles may either contain one main type of fiber or be composed of numerous types in various combinations. This variability gives muscle a great deal of flexibility in its ability to generate force and movement.

It appears that the neural impulse determines the fiber type developmentally. Chronic, slow neural impulses produce slow-twitch fibers while faster impulses result in the development of fast-twitch fibers. However, muscle has a great deal of plasticity and fiber type can change even in adult muscle. For example, cross-reinnervation

Table 1. Myosin heavy chain proteins detected in rodents and their expression patterns.

\begin{tabular}{|l|l|l|}
\hline Genes & Proteins & Expression pattern \\
\hline MYH2 & MyHC-2A & Fast type 2A fibers \\
\hline MYH1 & MyHC-2X & Fast type 2X fibers \\
\hline MYH4 & MyHC-2B & Fast type 2B fibers \\
\hline MYH7 & MyHC- $\beta$ & Heart and slow muscles \\
\hline$M Y H 3$ & MyHC-emb & Developing Muscle \\
\hline
\end{tabular}

can change fiber type: fast muscles convert to slow when reinnervated with a slow nerve and slow muscles become fast when reinnervated by a fast nerve. ${ }^{4,5}$ With application of electrical stimulation, chronic slow frequency stimulation of denervated slow muscles can maintain a slow fiber phenotype. Chronic slow frequency stimulation of denervated muscles expressing mainly fast-twitch fibers can switch to a slow fiber type and vice versa. $^{4,5}$ Human skeletal muscle contains three main types of fibers: Type 1,2A, and 2X. To date, only trace amounts of $2 \mathrm{~B}$ have been detected. The four main fiber types described above are found in rodents. In rats, the Gastrocnemius muscle contains a heterogeneous composition of fiber type, having both fast and slowtwitch fibers. ${ }^{6}$ The Extensor Digitorum Longus (EDL) muscle in rats is composed mainly of fast-twitch fibers, ${ }^{7}$ while the Soleus (SOL) muscle contains mainly slowtwitch fibers. ${ }^{8}$

\section{ERGIA}

The ether-a-gogo related gene-1a (Ergla) encodes a potassium channel that is partially responsible for repolarization of the cardiac action potential. ${ }^{9-11}$ Numerous alternative splice variants have been reported, but only the ERGIA and ERGIB gene products are detected in cardiac tissue. ${ }^{11,12}$ The ERG1A protein has also been reported in the brain, smooth muscle of numerous mammalian species, and various cancer cells. ${ }^{9}, 11$ The $1 \mathrm{~B}$ variant is considered to be a cardiac specific isoform, ${ }^{13,14}$ although there are reports of its detection in brain and cancer cells. ${ }^{11,12}$ Interestingly, it has been shown in rodents that the ERG1A $\mathrm{K}^{+}$channel variant is upregulated in skeletal muscle atrophying in response to either disuse or cancer cachexia. ${ }^{15}$ Skeletal muscle atrophy may occur with many different diseases (e.g., cancer cachexia, HIV/AIDS, and diabetes), injury (e.g. spinal cord damage or denervation), disuse, and fasting; it also occurs with normal aging and during space flight. ${ }^{16-20}$ Skeletal muscle atrophy may be quite debilitating. For example, although atrophy of leg muscles may reach a steady state in terms of muscle loss (e.g., in thoracic level spinal cord injury), ${ }^{21}$ it may progress to complete loss of muscle contractile proteins with misplaced groups of myonuclei when leg muscles are permanently denervated (as in complete Conus Cauda Syndrome). ${ }^{22,23}$ Further, muscle protein loss may be particularly severe in normally innervated muscles (e.g., in tumor cachectic syndromes related or unrelated to chemotherapy). ${ }^{24,25}$ Muscle atrophy occurs when there is an imbalance in protein degradation and protein synthesis that results in a net loss of muscle protein. The majority of muscle degradation results from the activity of three proteolytic systems: calpains, cathepsins, and the ubiquitin proteasome pathway (UPP). ${ }^{26-28}$ It has been reported that, when Ergla is ectopically expressed in the Gastrocnemius muscles of mice, abundance of the UPP E3 ligase MuRF1 is increased, UPP activity is increased, and myofiber size decreases significantly. ${ }^{15}$ The data 


\section{ERG1A potassium channel in skeletal muscle fibers}

Eur J Transl Myol 29 (3): 235-245, 2019

suggest that ERG1A plays a role in the mechanism(s) producing skeletal muscle atrophy.

\section{Hypothesis}

ERG1A is an integral membrane voltage-gated potassium channel and has the potential to affect membrane characteristics and thus ultimately the speed of muscle contraction. Therefore, we hypothesized that ERG1A abundance could be fiber type dependent, varying between the slow and fast twitch fiber types. To test this hypothesis, we first used immunohistochemistry to measure ERG1A fluorescence in the muscle fibers of both the SOL and EDL muscles. We intended to compare ERG1A fluorescence per fiber type within a muscle; however, because the EDL and SOL muscles are nearly homogeneous in terms of fiber type composition, we were unable to do so. Nonetheless, we did discover that ERG1A is more abundant in SOL muscle, which contains predominantly slow-type fibers, than in the EDL muscle, which contains predominantly fast-type fibers. These results were consistent across immunoblots of muscles from old and young rats. To look at ERG1A abundance across fiber type composition, we needed to test ERG1A abundance in a muscle that had a more heterogeneous composition of fiber types. Thus, we used immunohistochemistry (IHC) to co-immunostain Gastrocnemius muscle sections for ERG1A and a fiber type specific MyHC protein (slow-twitch or fast-twitch). We then measured ERG1A fluorescence in each fiber type to determine if there was a difference in abundance of the ERG1A K ${ }^{+}$channel between slow-twitch and fasttwitch muscle fibers.

\section{Materials and Methods}

Animals.

All procedures were approved by the SIU Animal Care and Use Committee. Soleus, Extensor Digitorum Longus, and Gastrocnemius muscles from FDB rats (NIH; Bethesda, MD) at both 3 and 30 months of age were generously provided by Dr. Don Torry (SIU School of Medicine).

\section{Cryo-sectioning}

Muscles were embedded in Optimal Cutting Temperature (OCT) Cryo-sectioning Compound (Tissue-Tek; Lakewood, FL) and cross sectioned at $20 \mu \mathrm{m}$ using a cryostat microtome (Leica; Norcross, GA).

\section{Immunohistochemistry (IHC)}

Muscle sections were not fixed. They were incubated with $3 \%$ hydrogen peroxide (15 minutes at room temperature [RT]) and then rinsed with phosphate buffered saline (PBS). Next, they were dipped in $0.3 \%$ Sudan Black (to quench autofluorescence) in PBS containing $0.5 \%$ triton $\mathrm{X}-100$ for 30 seconds and then rinsed with PBS. The sections were incubated in blocking buffer (5\% normal goat serum [NGS] $+2 \%$ TritonX-100 in PBS) for one hour and then incubated with either anti-slow or anti-fast $\mathrm{MyHC}$ mouse primary antibody at room temperature also for an hour. One section per muscle was not incubated with the primary antibody as control. The sections were then rinsed with PBS. Non-control sections were incubated in an in-house anti-ERG1A primary antibody ${ }^{29}$ overnight. After incubation, the sections were rinsed and secondary antibodies (goat anti-mouse IgG labelled with Alexafluor 568 [Sigma; St. Louis, MO] and goat anti-rabbit IgG conjugated with HRP-tyramide Alexa-fluor 488 [Invitrogen; Waltham, MA]) were mixed and applied to all sections, including controls, at room temperature for 1 hour. Sections were then rinsed with PBS. The ERG1A signal was developed using a Tyramide kit (per manufacturer's instructions) for $5 \mathrm{~min}$.

\section{Imaging}

Images were acquired using a Leica DM4500 microscope with a Leica DFC 340FX camera. At least two fields per section, with three sections per muscle were photographed and acquisition parameters were maintained identically across samples. ERG1A (green) fluorescence in muscle fibers stained for either slow or fast $\mathrm{MyHC}$ (red) was measured as single-pixel brightness.

\section{ImageJ analysis}

Using ImageJ software, single-pixel brightness measurements were taken from the sarcolemma of complete fibers that were also stained for the desired type of muscle fiber (fast or slow). Brightness values were recorded as integers ranging from 0 (no signal) to 256 (white) and the average brightness value ( \pm standard error of the mean [SEM]) for each section was determined.

\section{Homogenization}

All procedures were carried out on ice and all buffers contained a mixture of protease inhibitors (1 $\mathrm{mM}$ phenylmethanesulfonylfluoride [PMSF], $2 \mu \mathrm{M}$ pepstatin, $1 \mathrm{mM}$ benzamidine, $8 \mu \mathrm{g} / \mathrm{ml}$ aprotinin, $1 \mathrm{mM}$ iodoacetamide, $1 \mathrm{mM}$ 1,10-phenanthroline, $1 \mu \mathrm{g} / \mathrm{ml}$ leupeptin). Approximately $1 \mathrm{~g}$ of muscle was homogenized in $10 \mathrm{ml}$ Tris-EDTA (TE) buffer $(10 \mathrm{mM}$ Tris, $1 \mathrm{mM}$ EDTA, pH 7.5) on ice. Nuclei and cell debris were removed by centrifugation $(1,000 \times \mathrm{g}, 10 \mathrm{~min})$. The first supernatant was collected and the pellet was resuspended with $8 \mathrm{ml}$ TE buffer and centrifuged (1,000 $\times \mathrm{g}$ for $8 \mathrm{~min}$ ). The supernatants were pooled and centrifuged at $40,000 \times \mathrm{g}$ for $10 \mathrm{~min}$. Supernatants were poured off and the pellets were resuspended in $15 \mathrm{ml}$ of homogenization buffer (TE plus protease inhibitors as described above) containing 0.6 M potassium iodide (a chaotropic agent), and incubated on ice for $30 \mathrm{~min}$. The suspension was then centrifuged at $40,000 \times \mathrm{g}$ at $4^{\circ} \mathrm{C}$ for $10 \mathrm{~min}$. This pellet was then resuspended in TE (as above) by vortexing and then centrifuged at 40,000 $\times \mathrm{g}$ at $4^{\circ} \mathrm{C}$ for $10 \mathrm{~min}$ two times to remove the potassium iodide. Finally, the pellet was resuspended in an 


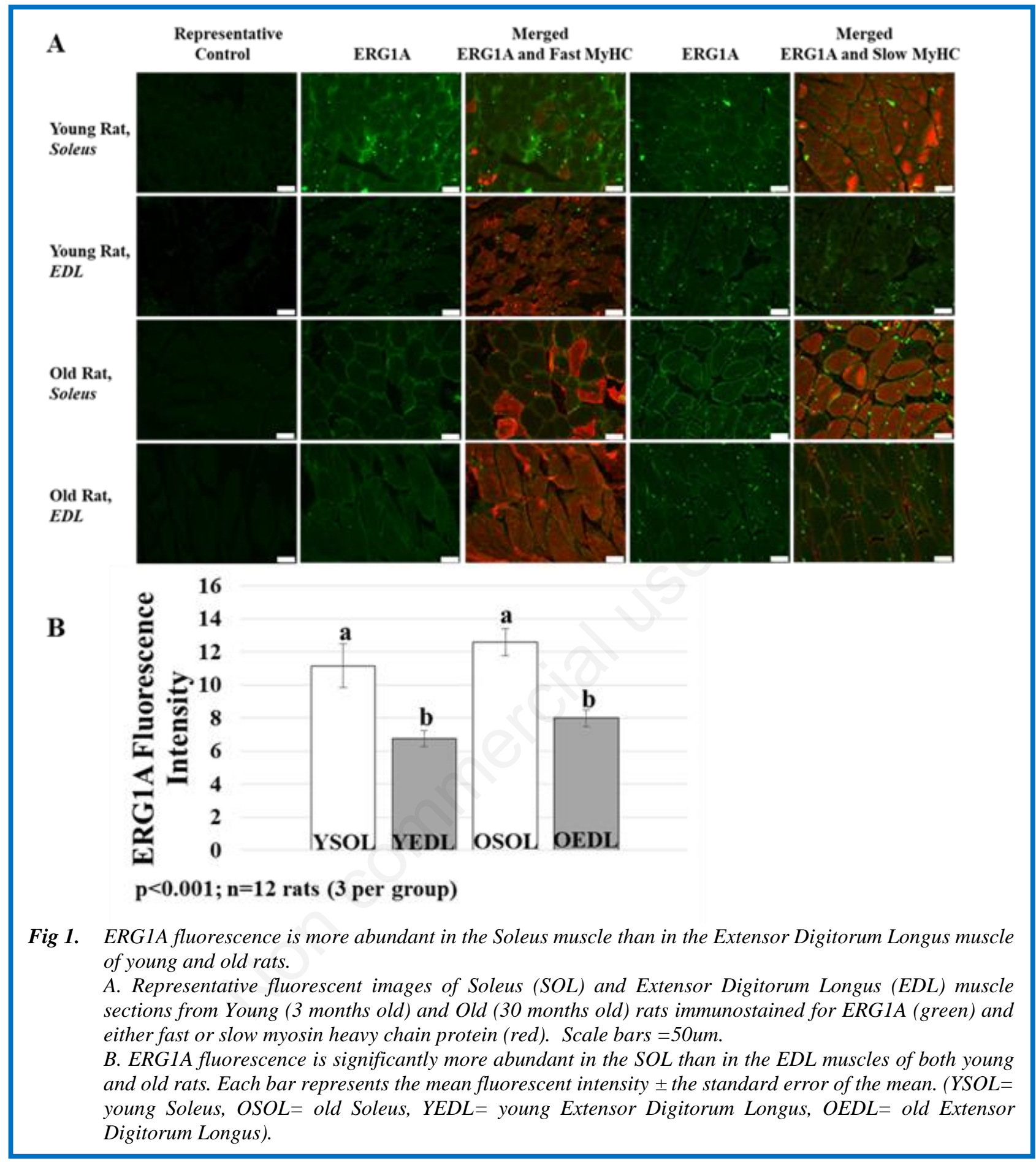

appropriate amount of solubilization buffer (TE buffer as described above with $2 \%$ Triton X-100), incubated on ice for 1 hour and finally centrifuged at $17,400 \times \mathrm{g}$ at $4^{\circ} \mathrm{C}$ for $30 \mathrm{~min}$ to pellet insoluble materials. Protein assays (BioRad DC kit; Hercules, CA) were performed according to manufacturer directions, and the samples were aliquoted and frozen at $-80^{\circ} \mathrm{C} .^{27}$

\section{Immunoblot}

Polyacrylamide SDS gels (separating gel $7.5 \%$ and stacking gel $4 \%$ acrylamide) were used with each electrophoresis experiment. Aliquots of sample protein (indicated in each figure) were combined with sample diluting buffer $(0.3 \mathrm{M}$ Tris, $50 \%$ glycerol, $5 \%$ SDS, $0.5 \mathrm{M}$ dithiothreitol [DTT], and 0.2\% Bromophenol Blue) and boiled for five minutes. The gel was then loaded with standard (BioRad Kaleidoscope Precision Plus Protein Standards; BioRad, Hercules, CA; $12 \mu \mathrm{l}$ ) and samples and these were electrophoresed at $200 \mathrm{~V}$ until the dye front exited the gel. The proteins were then transferred to PVDF membrane (BioRad, Hercules, CA; $0.2 \mu \mathrm{M}$ pore 


\section{A. Old Rat Muscle}

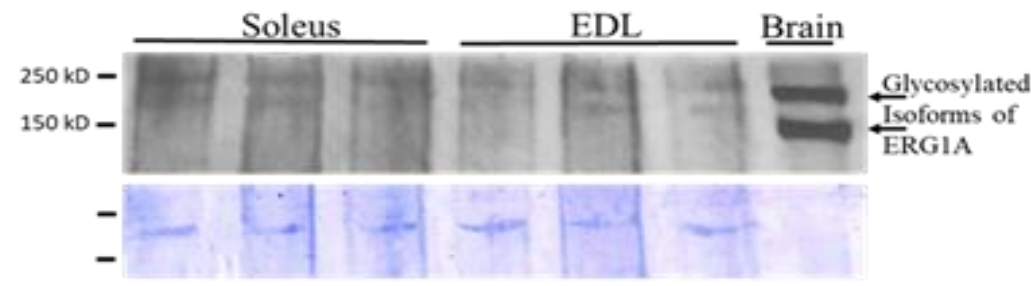

B. Young Rat Muscle

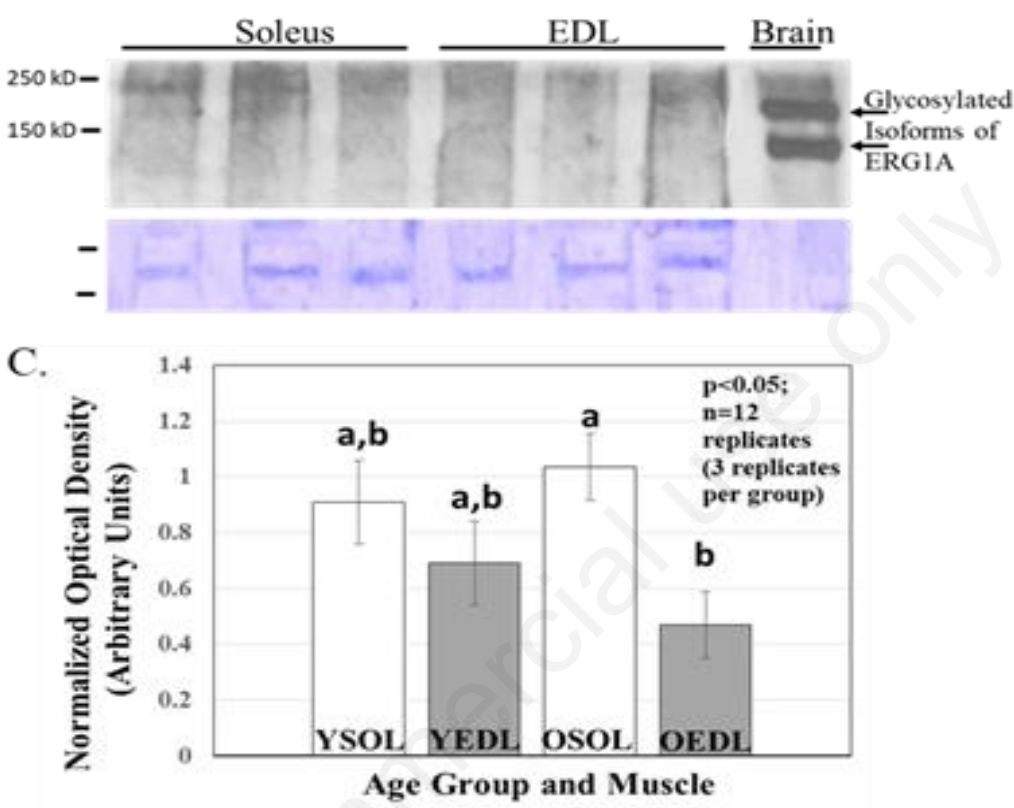

Fig 2. ERG1A protein is more abundant in the Soleus muscle than in the Extensor Digitorum Longus muscle of young and old rats.

A. Immunoblot of Soleus (SOL) and Extensor Digitorum Longus (EDL) muscles from 30-month-old rats. Skeletal muscle homogenate (70 $\mu \mathrm{g}$ protein) was loaded into each lane. Coomassie stained membrane below blot (in blue) confirms that an equal amount of skeletal muscle homogenate were loaded into each lane of the gel.

B. Immunoblot of Soleus (SOL) and Extensor Digitorum Longus (EDL) muscles from 3-month-old rats. Skeletal muscle homogenate (70 $\mu \mathrm{g}$ protein) was loaded into each lane. Coomassie stained membrane below blot (in blue) confirms that an equal amount of skeletal muscle homogenate were loaded into each lane of the gel.

C. ERGIA protein is more abundant in the Soleus (Sol) than in the Extensor Digitorum Longus (EDL) muscle and this difference is statistically significant in the old rat skeletal muscle. The optical densities of the two glycosylated ERGIA bands were combined per rat and divided by the brain sample optical density (the same brain sample [15 $\mu \mathrm{g}$ protein] was used in both immunoblots) to give a ratio which normalized the data for comparison across immunoblots. The bar graph depicts mean normalized optical densities \pm standard error of the mean.

size). After transfer, the membrane was rinsed in PBS 3 times for five minutes each rinse. The sample-containing membrane was then incubated in blocking buffer $(0.2 \%$ I-Block [Applied Biosystems; Foster City, CA] containing $0.1 \%$ Tween-20 in $1 \mathrm{X}$ PBS, $\mathrm{pH} 7.4$ ) and rocked for one hour. The necessary primary antibody was diluted (per figure legends) in a second blocking buffer
(5\% NGS, $0.2 \%$ Triton $\mathrm{X}-100,0.1 \%$ sodium azide in PBS) and the primary antibody was placed on top of the membrane and incubated at $4^{\circ} \mathrm{C}$ in a wet chamber overnight. The next day the membranes were removed from primary antibody and rocked for 10 minutes each in two changes of I-block buffer $(0.2 \%$ I-Block and $0.1 \%$ Tween-20 in $1 \mathrm{X}$ PBS). It was then removed from the 


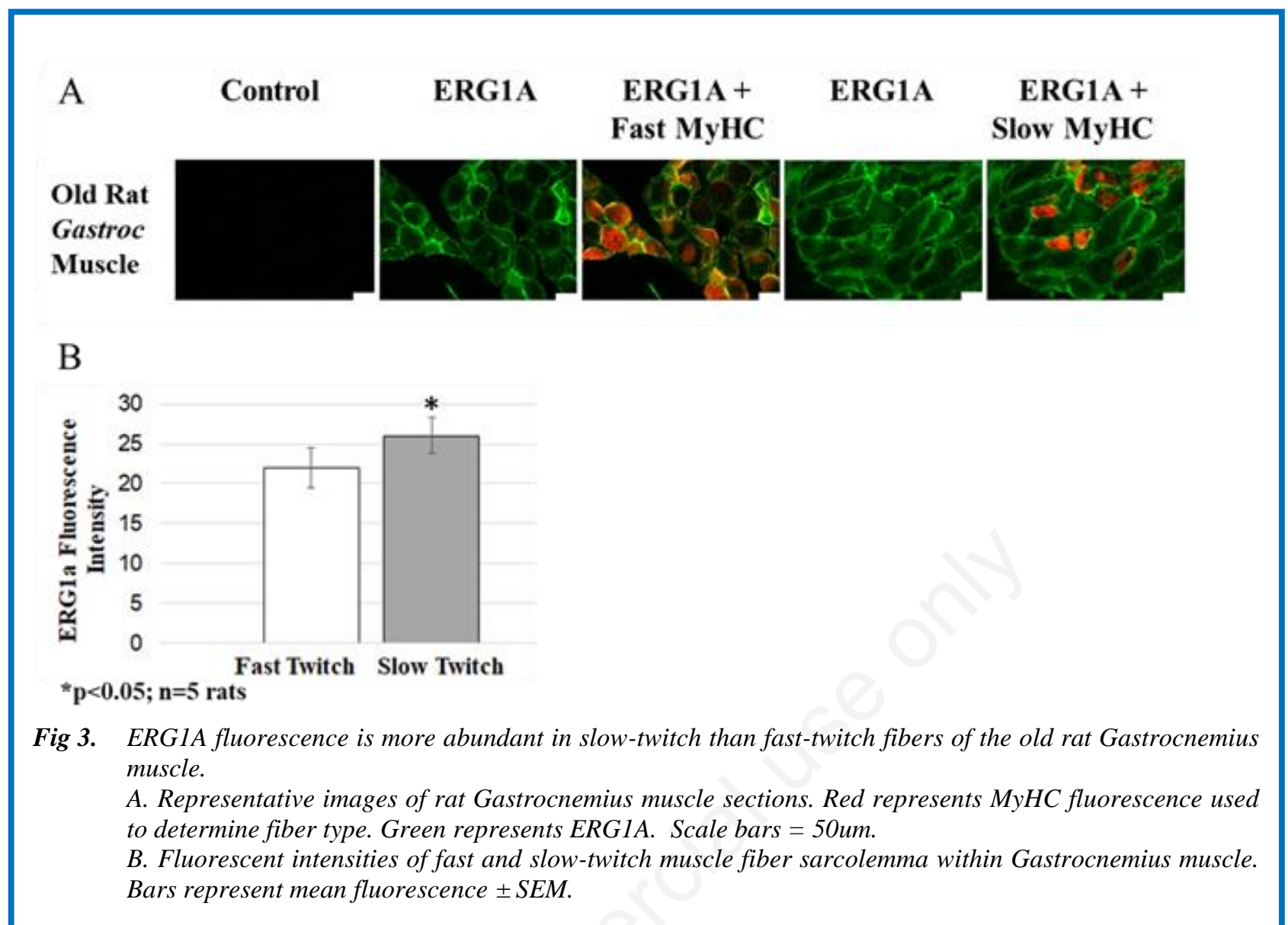

buffer and incubated in a goat anti-rabbit alkaline phosphatase (AP)-conjugated secondary antibody (diluted 1:1000 in I-block buffer) for 1 hour. The secondary antibody was removed and the membranes were washed three times for 15 minutes in I-block buffer. The membrane was then washed twice in assay buffer (0.1\% Tween 20 in Tris-buffered saline [TBS]). Membranes were then laid on a plastic block and ImmunStar AP substrate (BioRad, Hercules, CA) at $37^{\circ} \mathrm{C}$ was applied to the membranes for five minutes. These were then exposed to film and developed using an SRX-101A film processor (Konica Minolta, Chiyoda, Tokyo, Japan). After films were developed, they were scanned into a computer and ImageJ was used to measure the optical density (OD) of each band (Figure 1). For figure 2, the ODs of the two glycosylated ERG1A bands were combined per rat and divided by the brain sample OD (used in both immunoblots) to give a ratio which normalized the data for comparison across immunoblots.

\section{Statistics}

Both the single point brightness data from the immunohistochemistry (Figure 1) and the OD data from the immunoblot (muscle to brain OD ratio; Figure 2) were analyzed using a two-way ANOVA comparing by both age (young vs. old) and muscle (EDL vs. SOL). The interaction between age and muscle was not significant, and so the interaction was not included in the final model. When significant differences were found, means were compared using least squares means. To compare differences in brightness values of slow and fast fibers (Figure 3), the mean single point brightness value of the fast fiber was subtracted from that of the slow fiber within the Gastrocnemius muscle per rat. A paired Student's t-test was used to compare this difference to zero (representing no difference). Immunoblot data from the Gastrocnemius muscles (Figure 4) were analyzed using a Student's t-test. All statistical tests were completed in SAS 9.4 (SAS Institute, Cary, NC). The ANOVA analyses were completed using the General Linear Model Procedure of SAS. All p values equal to or less than 0.051 are considered statistically significant.

\section{Results}

ERG1A Fluorescence is More Intense in Soleus than Extensor Digitorum Longus Muscles of Rats

IHC reveals that ERG1A fluorescence intensity, as detected in the muscle fiber sarcolemma, is on average $39.4 \%$ greater $(\mathrm{p}<0.001 ; \mathrm{n}=12$ rats $)$ in the SOL than in the EDL muscles of young rats and $36.5 \%$ greater ( $\mathrm{p}<0.001 ; \mathrm{n}=12$ rats) in the SOL than in the EDL muscles of old rats (Fig. 1 A, B). There is no significant difference in ERG1A fluorescence intensity that can be attributed to age in these muscles; however, there is an 

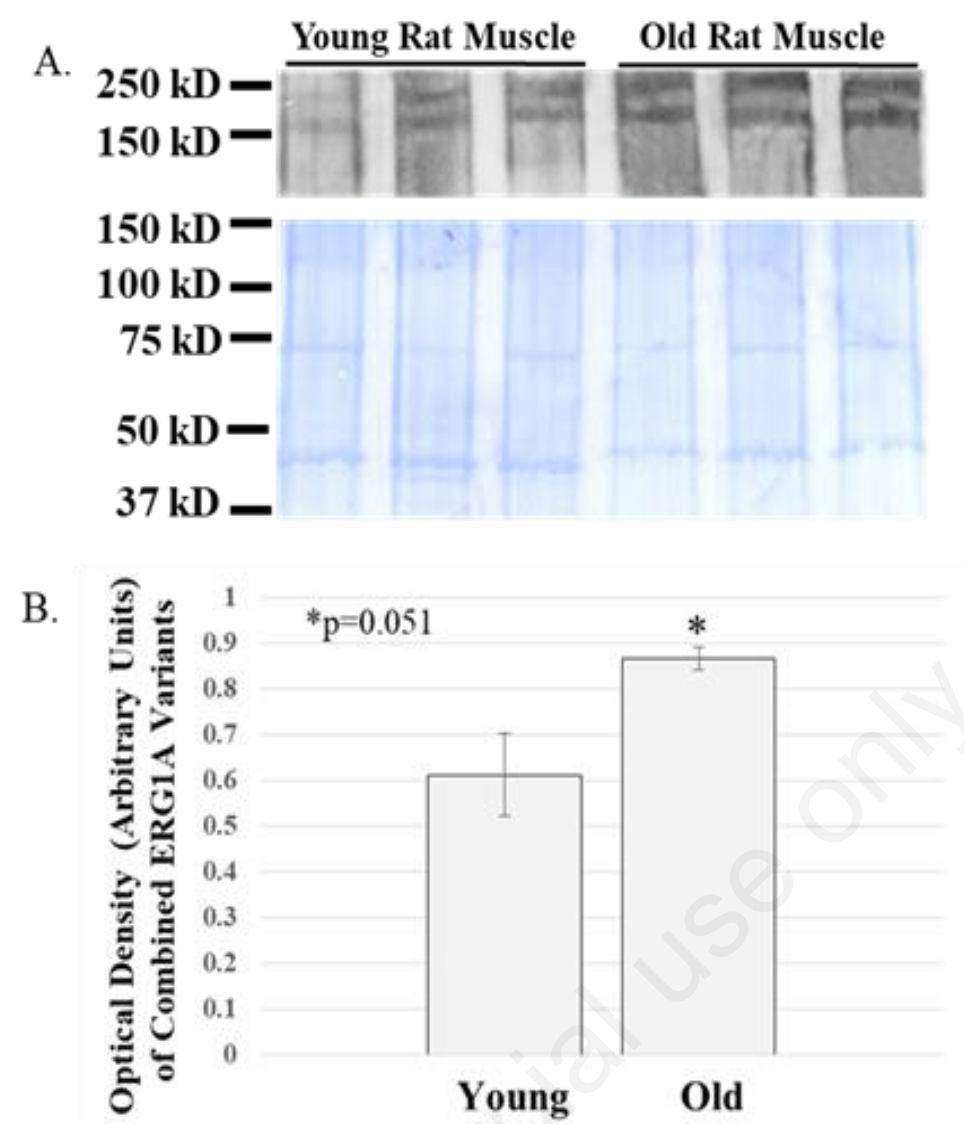

Fig 4. ERG1A protein is more abundant in old than young rat Gastrocnemius muscle.

A. Immunoblot of Gastrocnemius muscle homogenate from young and old rats. Muscle homogenate (70 $\mu \mathrm{g}$ protein) was loaded into each well. Coomassie stained membrane confirms that equal amounts of protein homogenate were loaded into each lane of the gel. (From left to right: Wells 1-3= young rat, wells 4-6=old rat.)

B. ERG1A is more abundant in the Gastrocnemius muscle of old than young rat. The optical densities of the two glycosylated ERGIA bands were combined per rat for statistical analysis. Bar graph depicts mean combined optical densities \pm standard error of the mean.

observed tendency for intensity to be greater in the muscle from the older animals. Immunoblots were also performed and the data show that, similarly to the IHC data, ERG1A protein is a significant $48.3 \%$ more abundant in SOL than in EDL muscles of old rats while it is $24.0 \%$ more abundant $(\mathrm{p}<0.05 ; \mathrm{n}=12)$ in SOL than in EDL muscles of young rats (Fig. 2A-C).

ERG1A Fluorescence is More Abundant in Slow-Twitch than Fast-Twitch Fibers of the Rat Gastrocnemius

Using IHC, we determined that ERG1A is more abundant in slow-twitch fibers than in fast-twitch fibers of rat Gastrocnemius muscle (Fig. 3 A, B). Specifically, we found that, on average, fluorescent intensity is $17.2 \%$ greater in slow-twitch than in fast-twitch fibers within each rat $(p<0.05, n=5)$. This suggests that the results of the SOL and EDL experiments may be caused by differences in ERG1A abundance within fiber type rather than/or in addition to within muscle type.
ERG1A is More Abundant in the Gastrocnemius Muscles of Old than Young Rats

Using optical density measurements of immunoblots (Figure 4), we were able to determine that, on average, ERG1A is $41.6 \%(\mathrm{p}=0.051 ; \mathrm{n}=6)$ more abundant in old than in young rat Gastrocnemius muscles.

\section{Discussion}

The ERG1A $\mathrm{K}^{+}$channel is known to contribute to repolarization of the action potential in heart. It was not detected in skeletal muscle until the Pond and Hannon labs reported finding it in the atrophying skeletal muscle of unweighted and cachectic mice. ${ }^{15}$ These laboratories also reported that ectopic expression of mouse Ergla (Merg Ia) would increase UPP activity in skeletal muscle, while both genetic and pharmacological block of the MERG1A channel would inhibit the loss of muscle in hind limb unweighted mice. ${ }^{15}$ Further, they reported that ectopic expression of Mergla would enhance the 
abundance of the UPP E3 ligase, MuRF1, ${ }^{15}$ suggesting that the increase in this protein contributes to the enhanced UPP activity. Interestingly, the expression of Mergla did not affect expression nor abundance of the UPP E3 ligase atrophy marker, Mafbx/ATROGIN1. ${ }^{30}$ More work is needed to determine the role that the ERG1A $\mathrm{K}^{+}$channel plays in skeletal muscle physiology and atrophy.

\section{Role of ERG1a in "fast" and "slow" muscle fibers}

The terms "fast" and "slow" fibers refer to the speed of contraction of each fiber. Slow-twitch fibers reach peak contraction after approximately 110 milliseconds while fast-twitch reach peak contraction after only 40-60 milliseconds. ${ }^{31}$ These contractions are generated by different patterns of electrochemical impulses received from the connected neuron. Slow-twitch fibers receive impulses from their neurons at $10-50 \mathrm{~Hz}$, while fasttwitch fibers receive impulses ranging in frequency from 30 to more than $100 \mathrm{~Hz}^{32}$ Indeed differences in membrane characteristics exist slow and fast type fibers. When compared, fast-twitch fibers hold a higher resting membrane potential, have a greater amplitude of action potential, have higher depolarization and repolarization rates, and possess more variable patterns of depolarization. ${ }^{33}$ In cardiac tissue, ERG1A produces the $\mathrm{I}_{\mathrm{Kr}}$ current that contributes to repolarization of the action potential. ${ }^{34}$ Indeed, mutations of the ERG1 channel lead to enhanced repolarization, consequent prolongation of the action potential, and can lead to development of the cardiac disorder Long QT Syndrome 2. ${ }^{34}$ However, exactly how this channel affects skeletal muscle fiber membrane properties is unknown and should be explored. We suggest that the increase in Ergla gene expression and ERG1A protein function in skeletal muscle could increase the rate of repolarization of muscle fibers (as it does in the heart), thus decreasing the action potential duration (APD). A decreased APD would allow the cell to generate action potentials more quickly, emulating a firing pattern more typical of faster type II fibers. Indeed, slow-twitch fibers receive tonic low frequency impulses, while fast-twitch fibers receive phasic, high frequency impulses and, in atrophic muscle, it is reported that slow type fibers switch to fast-type fibers. ${ }^{5}$ It is possible that ERG1 contributes to this switch. However, the transition of slow to fast muscle fiber types, seems to be more related to the overall daily number of contractions of muscle fibers than to frequency of short activation bursts. ${ }^{35,36}$ Interestingly, the heart contracts more than 100,000 times a day, while the mean daily contraction for slow type muscle fibers in rodents is 20,000 times per day and 2,000 times for the fast-type muscle fibers. ${ }^{37,38,39}$ Whether these differences are relevant for the increased content of the ERG1A $\mathrm{K}^{+}$ channel in atrophic muscles of young and old animals remains to be determined.

It is possible that the upregulation of ERG1 $\mathrm{A} \mathrm{K}^{+}$channels could hyperpolarize the cells, increasing the stimulus necessary to depolarize the membrane. However, because the typical skeletal muscle cell resting membrane potential is so close to the reversal potential of $\mathrm{K}^{+}$, even if more channels were present and functional, the electrochemical gradient would likely prevent any large flux of $\mathrm{K}^{+}$across the membrane, thus preventing substantial hyperpolarization. Nonetheless, it is possible, but perhaps less likely, that there is an increase in the duration of action potential hyperpolarization, effectively increasing the relative refractory period and thus the time between action potentials. If this were true, then it would indeed require more stimulus for depolarization to occur and this could contribute to slowing of the muscle fiber firing. It is well-known that skeletal muscle speed of contraction slows as animals age, ${ }^{39}$ and, indeed, we find that ERG1A is significantly more abundant in old than in young rat Gastrocnemius muscle. Further, we noted a trend for ERG1A to be more abundant in both the SOL and EDL muscles of the old as compared to the young rats. With a larger sample size of rats, it is likely that the difference in ERG1A abundance between the SOL and EDL muscles of old and young rats would become statistically significant. Skeletal muscle naturally atrophies with normal aging, so it was suspected that we would find a greater abundance of ERG1A within the older rat muscle. It may well be that ERG1A contributes to the slower contraction of the old rat muscles. It has been shown in humans that, even in healthy aging, skeletal muscle that is retained by exercise becomes slower with an increase in slow-type fibers, which appear in clusters within skeletal muscle sections. ${ }^{39,40}$ Indeed, increased ERG1A channel abundance could be contributing to this remodeling. It is also possible that an increase in slow type fibers explains the increase in ERG1A channel protein in the older rat muscles. ${ }^{41}$

Could ERG1A affect fiber MyHC composition and thus fiber type?

Skeletal muscle fiber speed of contraction is mainly determined by the composition of $\mathrm{MyHC}$ in the sarcomere and $\mathrm{MyHC}$ composition is affected by energy demand and thus physical activity. In response to physical activity, muscle fiber type composition can switch, mainly converting from type II (fast twitch) to type I (slow twitch) fibers; mitochondrial biogenesis is often also increased. ${ }^{40,41}$ Muscle fiber response to physical activity is affected by the change in neural stimulus that accompanies the increased demand. Indeed, muscle fiber type composition is affected by the neural input received and cross reinnervation can change fiber type. ${ }^{38}$ That is, when a slow-twitch fiber is reinnervated to receive the neural impulse that would normally go to a fast-twitch fiber, the slow-twitch fiber will remodel, producing more fast MyHCs, and become a fast twitch fiber. Also, if a fast twitch fiber becomes reinnervated to receive a slower stimulus, then it may also "switch" and become a slow type fiber, synthesizing slower type MyHC. ${ }^{5}$ It is possible that ERG1A function contributes 
to this switch by affecting membrane properties and/or cellular signaling pathways. Further research is needed to determine the reason for this switching in atrophying muscle and of the potential affect ERG1A may have on skeletal muscle physiology.

\section{List of acronyms}

APD - action potential duration

EDL - Extensor Digitorum Longus

ERG1a - ether-a-go-go related gene- 1 a

IHC Immunohistochemistry

MyHC - Myosin heavy chain

NGS - normal goat serum

OCT - Optimal Cutting Temperature

OD - optical density

PBS - phosphate buffered saline

PMSF - phenylmethanesulfonylfluoride

RT - room temperature

SEM - standard error of the mean

SOL - Soleus

TE - Tris-EDTA

UPP - ubiquitin proteasome pathway

\section{Authors contributions}

LBA, OK, and BC are SIU undergraduate students working in the Pond laboratory. $\mathrm{CDL}$ and BHM are visiting undergraduate students working in the Pond lab for the summers. LBA cryosectioned muscle, immunostained and imaged skeletal muscle; prepared muscle homogenates and immunoblots; helped with data analysis; and wrote the manuscript. CDL analyzed the data and contributed to interpretation of the data and writing of the manuscript. OK sectioned muscle, collected data from immunostained sections, and reviewed the manuscript. BHM sectioned muscle, performed immunohistochemistry, and reviewed the manuscript. BC sectioned and immunostained muscle and reviewed the manuscript. ALP trained students, supervised all techniques, and revised the final manuscript.

\section{Acknowledgments}

The authors would like to thank histology lab technician, Stacy McGhee, for keeping the histology facility in working condition and for help with cryo-sectioning. This paper was presented in part at the Midwest Student Biomedical Research Forum, Omaha, NE, February 17, 2018.

\section{Funding}

The work was supported by a SIU REACH Award (to LBA) from the Southern Illinois University (SIU) Office of the Vice Chancellor for Research and by a SIU School of Medicine Seed Grant to ALP.

\section{Conflict of Interest}

The authors declare no potential conflict of interests.

\section{Ethical Publication Statement}

We confirm that we have read the Journal's position on issues involved in ethical publication and affirm that this report is consistent with those guidelines.

\section{Corresponding Author}

Amber L. Pond, 1135 Lincoln Drive, Life Sciences 3, Room 2080, Carbondale, IL 62902, Phone: 618-4531582, FAX: 618-453-1527

Email: apond@siumed.edu

\section{E-mails of co-authors}

Luke B. Anderson: lukebriananderson@gmail.com

Chase D. Latour: cdlatour@unc.edu

OmarKhader: omar.khader@siu.edu

Bryce H. Massey: bmassey2000@gmail.com

Brittan Cobb: brittan.a.cobb@gmail.com

\section{References}

1. Bottinelli R, Canepari M, Reggiani C. Stienen GJ. Myofibrillar ATPase activity during isometric contraction and isomyosin composition in rat single skinned muscle fibres. J Phsiol 1994;481:663-75. doi:10.1113/jphysiol.1994.sp020472.

2. Galler S, Hilber K, Pette D. Force responses following stepwise length changes of rat skeletal muscle fibre types." J Physiol 1996; 493:219-27. doi:10.1113/jphysiol.1996.sp021377.

3. Schiaffino S. Muscle fiber type diversity revealed by anti-myosin heavy chain antibodies. FEBS J 2018;285: 3688-94. doi:10.1111/febs.142.

4. Schiaffino S, Reggiani C. Fiber types in mammalian skeletal muscles. Physiol Rev 2011;91:1447-1531. doi:10.1152/physrev.00031.2010.

5. Liu J, Liang XJ, Gan ZJ. Transcriptional regulatory circuits controlling muscle fiber type switching. Sci China Life Sci 2015;58:321-7. doi:10.1007/s 11427-015-4833-4.

6. Chabowski A, Chatham JC, Tandon NN, et al. Fatty acid transport and FAT/CD36 are increased in red but not in white skeletal muscle of ZDF rats. Am J Physiol Endocrinol Metab 2006;291:E675-82. Epub 2006 May 9.

7. Close R. Properties of motor units in fast and slow skeletal muscles of the rat. J Physiol 1967;193:4555. doi:10.1113/jphysiol.1967.sp008342.

8. Schiaffino S. Relations between structure and function in rat skeletal muscle fibers. J Cell Biol 1970;47: 107-19. doi:10.1083/jcb.47.1.107.

9. Vandenberg JI, Perry MD, Perrin MJ, et al. HERG $\mathrm{K}^{+}$channels: structure, function, and clinical significance. . Physiol Rev 2012;92:1393-478. doi:10.1152/physrev.00036.2011.

10. Jones EM, Roti Roti EC, Wang J, et al. Cardiac IKr channels minimally comprise HERG $1 \mathrm{a}$ and $1 \mathrm{~b}$ subunits. J Biol Chem 2004;279:44690-4. doi:10.1074/jbc.m408344200. 
11. London B, Trudeau MC, Newton KP, et al. Two isoforms of the mouse ether-a-go-go related gene coassemble to form channels with properties similar to the rapidly activating component of the cardiac delayed rectifier $\mathrm{K}+$ current. Circ Res 1997;81:870-8. doi:10.1161/01.res.81.5.870.

12. Lees-Miller JP, Kondo C, Wang L, Duff HJ. Electrophysiological characterization of an alternatively processed ERG $\mathrm{K}+$ channel in mouse and human hearts. Circ Res 1997;81:719-26. doi:10.1161/01.res.81.5.719.

13. Andersen JL, Schiaffino S. Mismatch between myosin heavy chain MRNA and protein distribution in human skeletal muscle fibers. Am J Physiol 1997;272(6 Pt 1):C1881-9. doi:10.1152/ajpcell. 1997.272.6.c1881.

14. Andersen JL, Schjerling P, Saltin B. Muscle, genes and athletic performance. Sci Am 2000;283:48-55. doi:10.1038/scientificamerican0900-48.

15. Wang X, Hockerman GH, Green HW, et al. Merg1a $\mathrm{K}^{+}$channel induces skeletal muscle atrophy by activating the ubiquitin proteasome pathway. FASEB J 2006:20:1531-3. doi:10.1096/fj.05-53fje.

16. Baoge L, Van Den Steen E, Rimbaut S. et al. Treatment of skeletal muscle injury: a review. ISRN Orthopedics 2012, pp. 1-7. doi:10.5402/2012/ 689012.

17. Bonaldo P, Sandri M. Cellular and molecular mechanisms of muscle atrophy. Dis Model Mech 2012; 6:25-39. doi:10.1242/dmm.010389.

18. Frontera W, Ochala J. Skeletal muscle: a brief review of structure and function. Calcified Tissue Int 2014:183-95. doi:10.1007/s00223-014-9915-y.

19. Ma L, Chu W, Chai J, et al. ER stress and subsequent activated calpain play a pivotal Role in skeletal muscle wasting after severe burn injury. Plos One, 2017;12: 10. doi:10.1371/journal.pone. 0186128.

20. Smith IJ, Lecker SH, Hasselgren PO, et al. Calpain activity and muscle wasting in sepsis. Am J PhysiolEndoc M 2008;295:4. doi:10.1152/ajpendo.90226. 2008.

21. Kern H, Hofer C, Mödlin M, et al. Stable muscle atrophy in long-term paraplegics with complete upper motor neuron lesion from 3- to 20-year SCI. Spinal Cord 2008;46:293-304. Epub 2007 Oct 23.

22. Kern H, Carraro U, Adami N, et al. Home-based functional electrical stimulation rescues permanently denervated muscles in paraplegic patients with complete lower motor neuron lesion. Neurorehabil Neural Repair 2010;24:709-21. Epub 2010 May 11.

23. Carraro U, Kern H, Gava P, et al. Recovery from muscle weakness by exercise and FES: lessons from masters,active or sedentary seniors and SCI patients. Aging Clin Exp Res 2016 Sep;3:1-12. DOI:10.1007/s40520-016-0619-1.
24. Coletti D, Aulino P, Pigna E, et al. Spontaneous Physical Activity Downregulates Pax7 in Cancer Cachexia. Stem Cells Int 2016;2016:6729268. doi: 10.1155/2016/6729268. Epub 2015 Dec 20.

25. Barreto R, Mandili G, Witzmann FA, et al. Cancer and Chemotherapy Contribute to Muscle Loss by Activating Common Signaling Pathways. Front Physiol 2016;7:472 eCollection 2016.

26. Franch HA, Price SR. Molecular signaling pathways regulating muscle proteolysis during atrophy. Curr Opin Clin Nutr 2005;8:271-275., doi:10.1097/01.mco.00001605.01331.45.

27. Glass DJ. Signaling pathways that mediate skeletal muscle hypertrophy and atrophy. Nat Cell Biol 2003;5:87-90. doi:10.1038/ncb0203-87.

28. Kandarian SC, Jackman RW. Intracellular signaling during skeletal muscle atrophy. Muscle Nerve, vol. 2006;33:155-65. doi:10.1002/mus.20442.

29. Pond AL, Scheve BK, Benedict AT, et al. Expression of distinct ERG proteins in rat, mouse, and human Heart. J Biol Chem 2000;275:59976006. doi:10.1074/jbc.275.8.5997.

30. Hockerman GH, Dethrow NM, Hameed S, et al. The Ubr2 gene Is expressed in skeletal muscle atrophying as a result of hind limb suspension, but not merg1a expression alone. Eur J Transl Myol 2014;24:3. doi:10.4081/bam.2014.3.173.

31. Brooks GA, Fahey TD, Baldwin KM. Exercise physiology: human bioenergetics and its applications. 4th ed. Boston, MA: McGraw-Hill; 2005.

32. Boron WF, Boulpaep EL. Medical Physiology. Elsevier, 2017.

33. Wallinga WJ, Gielen F, Wirtz P, et al. The different intracellular action potentials of fast and slow muscle fibres. CORE, Electroencephalography and Clinical Neurophysiology 1985. core.ac.uk/display 192156162.

34. Curran M, Splawski I, Timothy KW, et al. A molecular basis for cardiac arrhythmia: HERG mutations cause long QT syndrome. Cell 1995;80:795-803.

35. Arpesella G, Carraro U, Mikus PM, et al. Activityrest stimulation of latissimus dorsi for cardiomyoplasty: 1-year results in sheep. Ann Thorac Surg 1998;66:1983-90.

36. Westgaard RH, Lømo T. Control of contractile properties within adaptive ranges by patterns of impulse activity in the rat. J Neurosci 1988;8:441526.

37. Hennig R, Lømo T. Firing patterns of motor units in normal rats. Nature 1985;20;314(6007):164-6.

38. Pette D, Vrbová G. What does chronic electrical stimulation teach us about muscle plasticity? Muscle Nerve 1999;22:666-77. doi:10.1002/(sici) 1097-4598(199906)22:63.0.co;2-z.

39. Mosole S, Carraro U, Kern H, et al. Long-term high-level exercise promotes muscle reinnervation 
ERG1A potassium channel in skeletal muscle fibers

Eur J Transl Myol 29 (3): 235-245, 2019

with age. J Neuropath Exp Neur 2014;73:284-94. doi:10.1097/nen.0000000000000032.

40. Chen G, Carroll S, Racay P et al. Deficiency in parvalbumin increases fatigue resistance in fasttwitch muscle and upregulates mitochondria. Am J Physiol-Cell PH 2001;281:C114-C122.

41. Paolini C, Quarta M, Nori A, et al. Reorganized stores and impaired calcium handling in skeletal muscle of mice lacking calsequestrin-1. J Physiol 2007;583:767-84.

Submission: July 15, 2019

Revision received: August 27, 2019

Acceptance: August 28, 2019 